\title{
Acoustics of the banjo: measurements and sound synthesis
}

\author{
Jim Woodhouse ${ }^{1, *}$, David Politzer ${ }^{2}$, and Hossein Mansour ${ }^{3}$ \\ ${ }^{1}$ Cambridge University Engineering Department, Trumpington Street, CB2 1PZ Cambridge, UK \\ ${ }^{2}$ 452-48 Caltech, Pasadena, 91125 CA, USA \\ ${ }^{3}$ Dassault Systèmes - SIMULIA, 5005 Wateridge Vista Dr., San Diego, 92121 CA, USA
}

Received 17 July 2020, Accepted 17 February 2021

\begin{abstract}
Measurements of vibrational response of an American 5-string banjo and of the sounds of played notes on the instrument are presented, and contrasted with corresponding results for a steel-string guitar. A synthesis model, fine-tuned using information from the measurements, has been used to investigate what acoustical features are necessary to produce recognisable banjo-like sound, and to explore the perceptual salience of a wide range of design modifications. Recognisable banjo sound seems to depend on the pattern of decay rates of "string modes", the loudness magnitude and profile, and a transient contribution to each played note from the "body modes". A formant-like feature, peaking around $500-800 \mathrm{~Hz}$ on the banjo tested, is found to play a key role. At higher frequencies the dynamic behaviour of the bridge produces additional formant-like features, reminiscent of the "bridge hill" of the violin, and these also produce clear perceptual effects.
\end{abstract}

Keywords: Banjo, Acoustics, Vibration, Synthesis

\section{Introduction}

Players of plucked-string musical instruments generally have a clear impression that different instruments have distinctive "voices". This is most obviously true between instruments of different types but also between different instruments of the same general type. A classical guitar is different from a harp, a harpsichord or a banjo, but also different guitars or banjos are different from each other. Furthermore, players commonly maintain that the voice of an instrument can be discerned whatever music is played on it. This seems familiar and obvious, but from the perspective of acoustics the final statement is somewhat surprising. Every acoustical stringed instrument has some kind of "soundboard", a resonant structure that allows the energy from the vibrating strings to couple effectively to sound waves in the surrounding air. The soundboard is most often a wooden structure, as in a guitar or a harpsichord, but many other materials may be used: the banjo uses a stretched membrane similar to a drum skin, while around the world stringed instruments can be found that employ gourds, tortoise shells and many other natural or man-made materials.

All these structures have one thing in common: they are vibrating systems with normal modes and natural

\section{*Corresponding author: jw12@cam.ac.uk}

The display of Audio files embedded in this PDF depends on the software used (PDF reader, video player, installed codec, direct display in the browser, etc.). Please see the Adobe Acrobat page for more explanation. You can also find the audio files at https://euphonics.org/ Chapter 5, Section 5.5. resonance frequencies, usually with fairly low damping (measured Q-factors typically fall in the approximate range 30-100, at least for wooden instruments: see for example Fig. 13 of [1]). As a result, the frequency response function characterising the transmission path between the string vibration and the radiated sound pressure will inevitably have large peaks and dips. A consequence of the peaky response of an acoustic instrument is that the distribution of sound energy between the overtones of a given plucked note will vary enormously from one note to another on the instrument. Can it really be the case that a particular instrument has a characteristic "voice" which is recognisable regardless of what music is played?

To begin to address such a broad question, it makes sense to look at extreme cases. The topic of this study is to investigate what makes the sound of a banjo distinctively different from other plucked-string instruments like the guitar. Even when strings, scale lengths, and pitches are chosen to be virtually identical, most listeners would agree that the sounds of banjo and guitar can be distinguished from just a few plucked notes. Measurements will be presented here of the vibration behaviour of banjo and guitar bodies and of the sounds produced during normal playing. These measurements will confirm that there are indeed large differences of vibrational behaviour between a banjo and an acoustical guitar: far bigger than the differences between guitars of a similar type, or between different banjos.

Once the driving-point frequency response of an instrument body has been measured at the bridge notch for a particular string, it is straightforward to combine this with 
known properties of the string itself, and synthesise fullycoupled transient response to plucking the string. Such synthesised responses will be used for two purposes here. They can be compared to measurements of the distribution of loss factors in frequency, and thus give a detailed interpretation of those measurements. They can also be turned into audio files, to find out whether listeners are convinced that they "sound like a banjo" or "sound like a guitar".

This project to explore the banjo has been driven throughout by listening to synthesised sounds based on a range of theoretical and numerical models. The models have been iterated until, in the opinion of the authors, they do indeed produce sounds that are convincingly banjo-like while accurately reflecting relevant aspects of the underlying physics. This background of interplay between theoretical understanding, model development and sounds makes the final story rather complicated to present. This paper will describe experimental results and synthesis methods, then introduce a set of sound demonstrations. A companion paper [2] will fill in the gaps in this description, with detailed analysis and modelling of the underlying physics. These models will be validated against the measurements presented in this paper, and they then allow additional synthesised sounds to be created. Some synthesised sounds are included in this paper, and a much larger collection of sounds has been provided via an accompanying web site [3]: the reader is encouraged to consult this web site alongside reading this paper.

Beyond the basic question of recognising a banjo among other instruments lie many details of interest to makers and players of instruments. Banjo enthusiasts, just like guitarists or violinists, are sensitive to many nuances of sound and playing behaviour of their instrument. Arguments rage about the merits of different constructional details and adjustments. Synthesis based on physical models can be used to explore the perceptual effects of changing model parameters, such as the tension in the banjo head membrane or the "break angle" of the strings over the bridge. There will be some discussion here, and more extensive discussion is given on the web site [3] which hosts the main set of sound files.

It should be emphasised that this paper and its companion do not include any formal psychoacoustical investigations. On the web site and in the two papers, opinions will be advanced about these sounds based on the authors' own listening, but readers can and should listen for themselves; they may or may not agree with those opinions. Of course, any such opinions need to be tested by careful perceptual studies. This paper and its companion provide some clear hypotheses, to be tested in future work: constraints of length do not allow such investigations to be included here.

Although there is extensive literature on the acoustics of the guitar, the violin, and other stringed instruments, there is rather little published work on the banjo [4-6]. These works shed some useful light on the physics of the instrument, but none of them examine all the consequences of using a membrane in place of a wooden box, or create a physical model capable of detailed synthesis of sounds to compare with real instruments, so there is relatively little overlap with the work to be described here and in the companion paper.

Furthermore, the existing literature on the guitar turns out to give rather little information about the particular issues to be raised here. Many authors seem to agree that the most important aspects of guitar acoustics concern a small number of low-frequency modes: their natural frequencies, mode shapes and sound radiation behaviour. There is some literature on numerical modelling that extends to higher frequency [7], but much of the literature is concentrated on measurements of mode shapes and sound fields, and increasingly elaborate numerical models: see for example [8-10]. The recent review article [11] gives many other literature references. Guitar makers can manipulate these low modes through constructional details, especially the chosen material, mass and bracing pattern for the soundboard $[12,13]$. There is some literature on perceptual assessment of differences between guitars, but again it is largely focussed on the influence of individual modes [14-17].

The individual body modes of a banjo are closely related to the well-studied case of drums (see for example [18-20]). The accompanying web site [3] contains a discussion of drums, and also (in section 5.3 of [3]) some measured examples of vibration modes of the banjo to be studied here. However, it will be suggested in the present work that individual low modes are less important for the banjo than in the case of the guitar: instead, several formant-like features will be revealed, and the sound examples provided on the accompanying web site [3] seem to suggest that these formants have higher perceptual salience than individual modes. The available evidence $[1,21]$ suggests that there are no comparable formant-like features in guitars, at least in those with glued bridges relevant to this study. However, a comparable feature, usually called the "bridge hill", has been extensively investigated in the violin [22-24]. This feature can be manipulated by violin makers, and is an important resource for tonal adjustment. The same is almost certainly true of the banjo formants.

\section{Banjo-guitar comparisons}

\subsection{The structure of the banjo}

The banjo is unusual among stringed instruments. Many of the elements responsible for the acoustical behaviour can be adjusted by the player. The tension of the head can be altered, and indeed the head membrane can be replaced; the bridge can be altered. No equivalent adjustments can be made on a fixed-bridge guitar: the player has to accept what the guitar maker gives them. Also in strong contrast to instruments like the guitar or the violin is the fact that the geometry of the resonant structure of a banjo is quite simple: a membrane is stretched across a circular frame (the "pot"), tensioned by a mechanism similar to that used in drums. Some banjos, including the main one tested here, have a metal ring (the "tone ring") between the pot and the membrane to control the boundary conditions at the edge of the membrane. As with most drums, the 

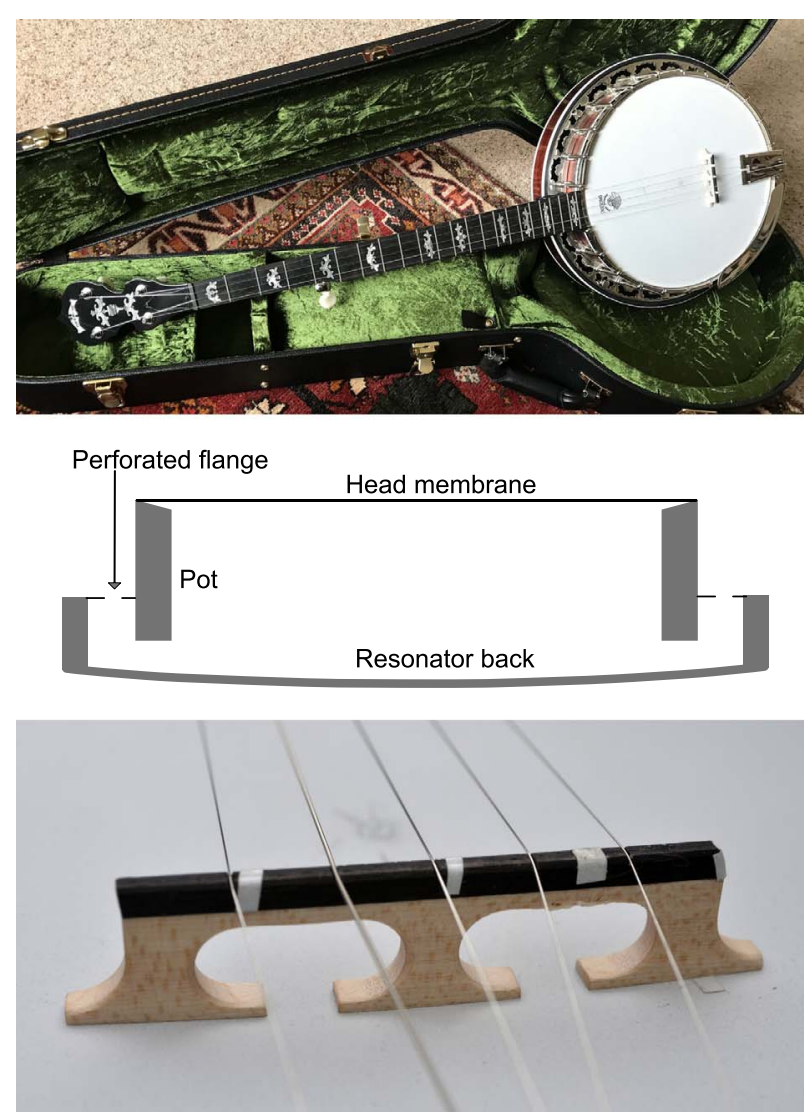

Figure 1. The Deering banjo, a schematic cross-section of the body with main components drawn approximately to scale, and a close-up of the bridge. The white patches on the bridge are reflective tape for the laser vibrometer measurements.

Table 1. Parameter values for the Deering banjo. Head tension is estimated from computer modelling: see the companion paper [2].

\begin{tabular}{lcc}
\hline Head diameter & $d$ & $268 \mathrm{~mm}$ \\
Head thickness & $h$ & $0.18 \mathrm{~mm}$ \\
Mass per unit area & $\sigma$ & $0.30 \mathrm{~kg} / \mathrm{m}^{2}$ \\
Tension & $T$ & $5.33 \mathrm{kN} / \mathrm{m}$ \\
Bridge mass & $m_{b}$ & $2.2 \mathrm{~g}$ \\
Bridge footprint & & $78 \times 5 \mathrm{~mm}$ \\
\hline
\end{tabular}

membrane was traditionally made of natural skin, but nowadays is usually made of a synthetic polymer: biaxially-oriented polyethylene terephthalate, more commonly known by trade names such as Mylar. The strings pass over a small "floating" bridge which rests on the membrane. The vibration of a circular stretched membrane is, of course, a well-understood problem going back at least as far as Rayleigh [18].

The main 5-string banjo used for measurements in this study is a Deering Eagle II, in the condition in which it was received from the manufacturer [25]. Figure 1 shows the banjo, and a close-up of its bridge. Some parameter values for this banjo and the strings with which it is fitted are given in Tables 1 and 2. The banjo has a removable
Table 2. Parameter values for the strings of the Deering banjo.

\begin{tabular}{lccccc}
\hline String & 1 & 2 & 3 & 4 & 5 \\
\hline Diameter $(\mathrm{mm})$ & 0.25 & 0.28 & 0.33 & 0.53 & 0.24 \\
Length $(\mathrm{m})$ & 0.67 & 0.67 & 0.67 & 0.67 & 0.50 \\
Frequency $(\mathrm{Hz})$ & 293.7 & 246.9 & 196.0 & 146.8 & 392.0 \\
Tension $(\mathrm{kg})$ & 6.19 & 5.29 & 4.66 & 5.76 & 5.56 \\
Impedance $(\mathrm{Ns} / \mathrm{m})$ & 0.154 & 0.157 & 0.174 & 0.287 & 0.139 \\
\hline
\end{tabular}

"resonator" back, which among other things modifies the sound radiation characteristics and provides a Helmholtz resonance at low frequency. Figure 1 includes an approximate scale drawing of a cross-section through the body, showing the configuration of the pot and resonator back. Some results will also be shown for two other banjos. Detailed comparisons will be made with a guitar by Martin Woodhouse [26]. This guitar, somewhat unusually for a steel-strung instrument, embodies a version of Torres-like fan bracing.

\subsection{Spectrograms and loudness profiles}

Since the aim is to understand the characteristic sound of a played banjo, it is useful to begin with some simple experimental comparisons between notes on the banjo and the guitar, based on normal playing. Conveniently, the top strings of the Deering banjo and the Woodhouse guitar are extremely similar: both are plain steel strings with the same diameter, and they are under very similar tensions. This allows a rather clean comparison between plucked notes on the two instruments. First, the note G4 $(392 \mathrm{~Hz})$ was plucked on both instruments: in standard tuning this corresponds to the 3rd fret on the guitar and the 5 th fret on the banjo. The notes were played in the normal way, and efforts were made to make them as similar as possible. The same plectrum was used, and the plucking distance from the bridge was $130 \mathrm{~mm}$ in both cases. The player sat in the same position with both instruments, and tried to use identical plucking gestures. The notes were recorded in a medium-sized domestic room suitable for chamber music, using a microphone (GRAS 46AR) placed on a stand above a carpeted floor, approximately $1 \mathrm{~m}$ away from the player.

Spectrograms of the two resulting sounds are shown in Figure 2, with identical scales including the colour scale indicating level. Both plots show the expected set of narrow vertical bands, associated with the near-harmonic overtones of the string in both cases. As can be seen particularly clearly for the guitar note, some overtones have significantly higher amplitude than others. This is in part because of a familiar effect arising from the chosen plucking point: it was roughly $1 / 5$ of the way along the string, so overtones 5 and 10 are relatively weak.

Careful inspection reveals some significant differences between the two plots. The banjo achieves higher levels than the guitar: the colour scale indicates decibels relative to the peak value for the banjo, and on this scale the peak value for the guitar was $-10.2 \mathrm{~dB}$. The decay times for the 

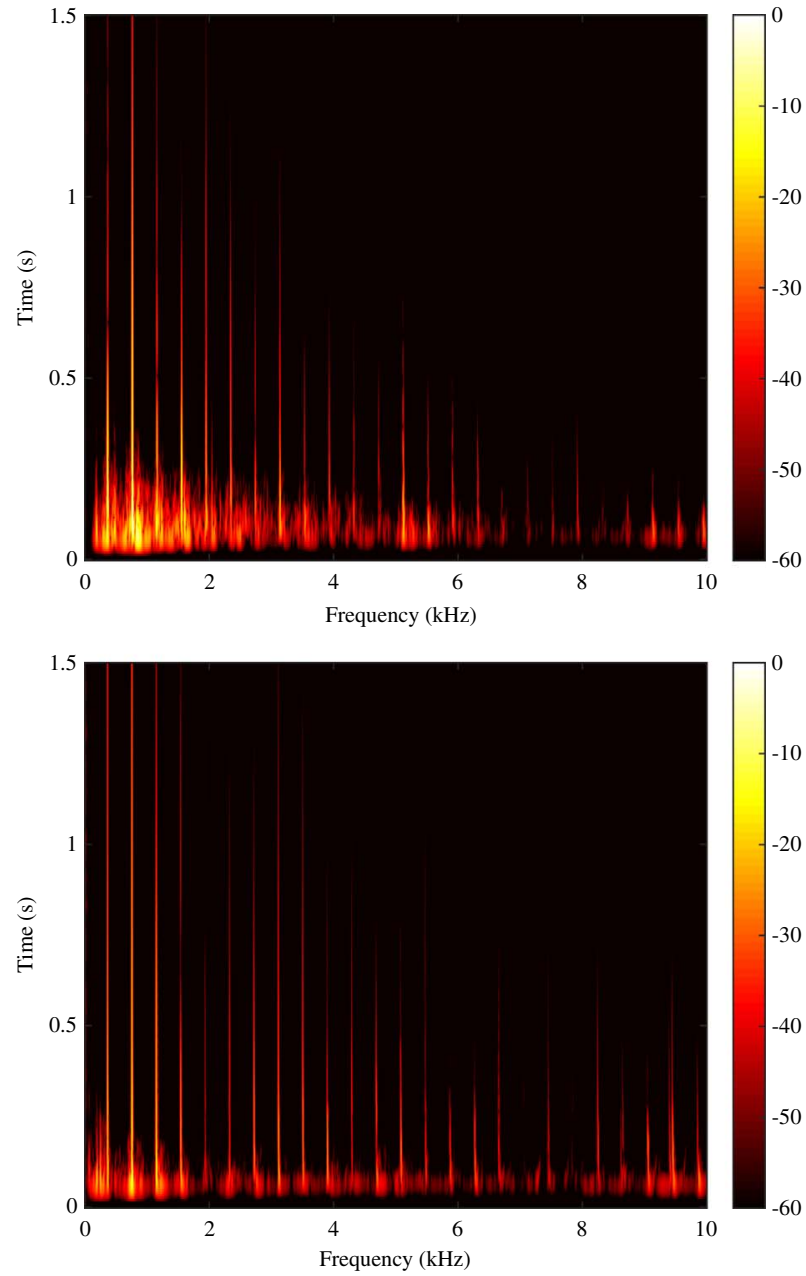

Figure 2. Spectrograms of plucked notes G4 $(392 \mathrm{~Hz})$ on the top string of (a) the banjo; (b) the guitar. The colour scale corresponds to identical levels (in $\mathrm{dB}$ ) in both plots.

overtones of the guitar string are generally significantly longer than for the banjo: a detailed quantitative analysis of decay times will be shown in Sections 2.3 and 3.2. For some overtones of the banjo string (for example the 1st and 3rd overtones), the colour can be seen to modulate in time, indicating beating between closely-spaced frequencies. This is probably caused by a frequency split between the two polarisations of string vibration, because they are differently affected by coupling to the banjo body. Both polarisations will normally be excited to some degree by a pluck.

Finally, there is a clear difference of appearance of the two spectrograms within the first $0.2 \mathrm{~s}$ or so after the pluck. The banjo shows a bright patch indicating a significant broad-band spread of radiated sound between the string overtones, extending up into the $\mathrm{kHz}$ region. For the guitar the corresponding activity between the string overtones is less conspicuous, and largely confined to lower frequencies. As will be explored in detail in Section 3.2, these broadband signals arise from transient excitation of modes of the coupled string-body system which have energy mainly in the body rather than in the string.

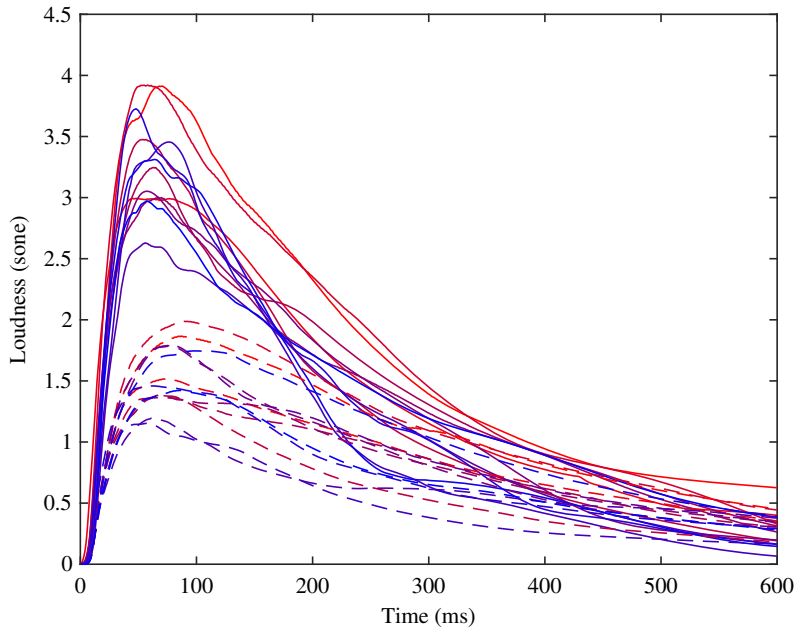

Figure 3. Short-term time-varying loudness computed from plectrum-plucked notes from the first octave on the top string of the banjo (solid lines) and the guitar (dashed lines).

A subjective observation can be mentioned at this stage. During the measurement of bridge admittance, to be described in Section 2.4, it was noted that the "clonk" noises of the miniature impulse hammer hitting the bridge (with the strings damped) were quite different for the banjo and the guitar. The guitar made a sound familiar from many other tests on wooden instruments: in general terms, guitars, lutes, violins, cellos and so on all make a rather similar sound during this testing. The banjo made a very different sound, reminiscent of a drum. This sound, arising from the transient excitation of modes of the body, is closely related to the feature just described in the spectrogram of a plucked note.

The spectrograms already give an indication, from the peak levels, that the banjo is louder than the guitar, a conclusion that does not come as a surprise. Nevertheless, it is worthwhile to analyse loudness of played notes on the two instruments in a quantitative way. With the same playing and recording setup just described, plectrum-played notes were recorded at every semitone from the open string up to the 12th fret, on both top strings. These were then processed by the procedure described by Moore et al. $[27,28]$, to calculate the short-term time-varying loudness in sones. The results are plotted in Figure 3: solid lines for the banjo and dashed lines for the guitar.

There are variations between individual notes on each instrument, partly caused by physical differences and partly, no doubt, by the inevitable variability of a human player. Nevertheless, the two groups of plots are quite distinct. The banjo notes are without exception louder than the guitar notes at early times following the pluck. They then show more rapid decay than the guitar notes, and the two clusters of curves merge into the same range after about $400 \mathrm{~ms}$. If the plot had been extended for a longer time, it would show the guitar notes eventually tending to be louder than the banjo notes in the tail of the decay. The differences revealed in this plot give the first clear 
evidence that banjo notes will be perceptually distinct from guitar notes (see for example Moore [29]).

Another subjective observation can be recorded, relating to these played notes. Although, as already noted, the two steel strings were in fact very similar, the impression to the player was that the guitar string felt "tighter" and less responsive. This impression is perhaps associated with the loudness difference: for a given plucking force applied by the player, the banjo made a louder sound than the guitar. If the player wanted to make sounds of similar loudness, more force would be needed on the guitar. Perhaps this leads to a subjective impression of unresponsiveness.

\subsection{Distribution of loss factors}

Loudness is, of course, a very crude measure of difference between musical sounds. For a more fine-grained view, the chromatic scales on the top strings of the banjo and guitar can be analysed in a different way. The detailed test procedure has been described in earlier papers [15, 30], and a related test methodology has been used by Fréour et al. [31]. Time-frequency analysis can be used to determine the best-fitted frequency and decay rate of every spectral peak satisfying a set of criteria to ensure data quality. The results can be plotted as a "cloud" of points to reveal patterns in the distribution of loss factors with frequency.

These measured results are plotted in Figure 4: guitar in black, banjo in red. Some areas of the plot have both red and black points, but elsewhere they tend to occupy rather different regions. The magenta line indicates the limit of applicability of the analysis method. Above this line the decay time becomes too short to be resolved, and absence of points in this region does not imply that no such combinations of frequency and loss factor exist in the real instruments. Lines to indicate the decay time constant are plotted in green dashes: details are given in the caption.

Detailed analysis of these results is deferred until Section 3.2, when comparisons with corresponding synthesised signals allow more illuminating interpretation. However, some simple observations can be made immediately. The black and red points lying a little below the magenta line at frequencies up to about $1 \mathrm{kHz}$ correspond to "body modes", already mentioned in connection with Figure 2. The "string modes", with an approximately harmonic sequence of frequencies based the fundamental of each played note, show lower loss factors. For these, the majority of the red points lie clearly higher than the black points, signalling slower decay for string modes of the guitar and faster decay for the banjo. The relevant decay time constants can be deduced by comparison with the green dashed lines: up to about $1 \mathrm{kHz}$, the banjo modes tend to have decay times in the range 100-200 ms, while the guitar modes lie in the range 300-400 ms. These values tie up well with the early decay times seen in the loudness profiles shown in Figure 3. The range of fundamental frequencies for the banjo notes was $294-588 \mathrm{~Hz}$, while that for the guitar notes was $330-660 \mathrm{~Hz}$, so the pattern revealed in the frequency range up to about $1 \mathrm{kHz}$ is mainly

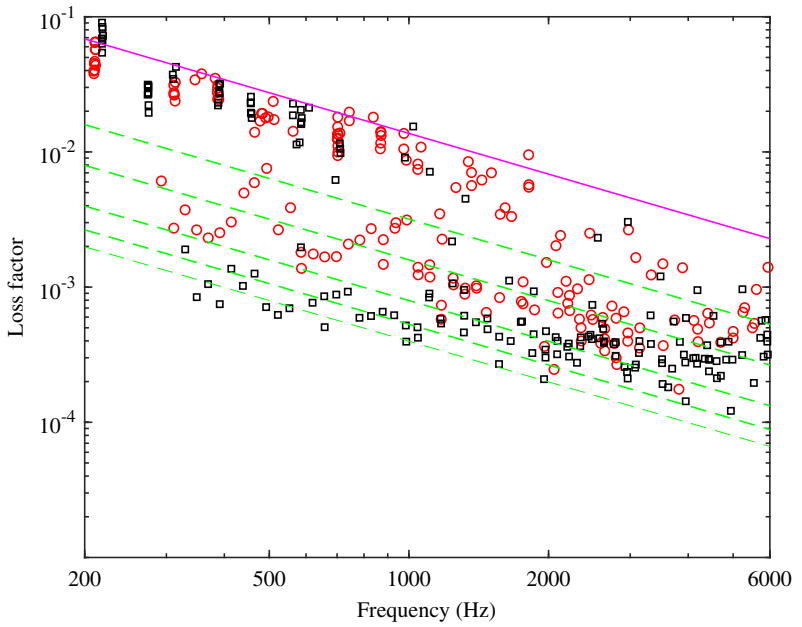

Figure 4. Loss factor versus frequency for modes excited by plucking the top string of the guitar (black squares) and the banjo (red circles). The analysis method cannot detect modes significantly above the magenta line. Green dashed lines indicate decay time constants $(\omega \eta)^{-1}=50 \mathrm{~ms}$ (top line), $100 \mathrm{~ms}, 200 \mathrm{~ms}$, $300 \mathrm{~ms}$ and $400 \mathrm{~ms}$ (lowest line).

associated with the fundamentals and second overtones of the played notes.

\subsection{Bridge admittance measurements}

To compare the structural vibration behaviour of the banjo and the Woodhouse guitar, the natural first step is to measure their input admittances at the bridge: the velocity response per unit force as a function of frequency. For any stringed instrument, this admittance characterises the dominant linear coupling between string and body, including the energy transfer rate from a string of given wave impedance. Measurements were made by the procedure described in earlier work [1]. Both instruments were tested with strings fitted and at normal playing tension. The strings were damped by weaving thin paper through them, without contacting the body or fingerboard. The instruments were supported in a vertical position with soft foam at the tailpiece end and a foam-lined clamp on the neck. Controlled force was applied to the bridge close to the notch for the first string, using a miniature impulse hammer (PCB 086D80) held in a pendulum fixture to ensure that the same point was struck every time. The resulting velocity response adjacent to the excitation point was measured with a laser-Doppler vibrometer (Polytec OFV-056). The signals were digitised into a $\mathrm{PC}$ at a sampling rate of $40 \mathrm{kHz}$, and processed with software written in Matlab. Each measurement involved at least 10 repeats, giving an averaged result for the frequency response function together with the associated coherence function (see for example McConnell and Varoto [32]).

Results are shown in Figure 5. The thick red line shows the admittance of the Deering banjo, while the thick blue line shows the Woodhouse guitar. The dashed red line shows the corresponding admittance of an inexpensive 


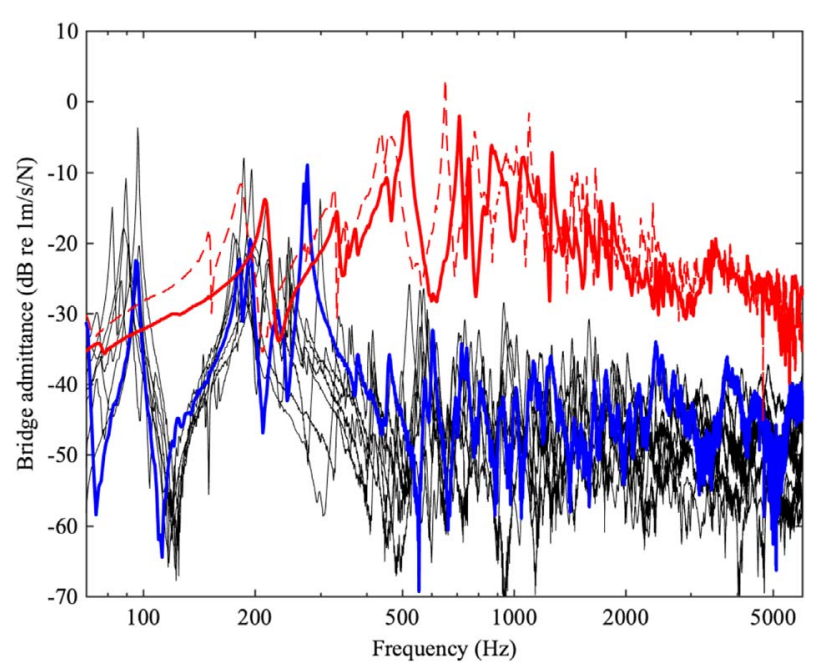

Figure 5. Measured drive-point admittance functions, all close to the bridge notch for the first string. Heavy red line: Deering banjo; dashed red line: Ozark banjo; heavy blue line: Woodhouse steel-string guitar; black lines: six other guitars of various types (see text).

banjo of Ozark brand. The cloud of thin black lines shows the admittances of a representative selection of six other guitars that are broadly comparable to the Woodhouse. The selection includes classical, flamenco and steel-string guitars; makers include Fleta, Ramirez, Smallman, O'Leary and Fylde; and the range includes fan-braced, lattice-braced and X-braced instruments.

The two banjos follow a very similar pattern, although the individual peaks for the Ozark are a little lower in frequency because it had a lower head tension than the Deering. The guitars also follow a very consistent pattern. Below about $300 \mathrm{~Hz}$ each guitar has a small number of strong peaks, and the varieties of construction lead to significant variations in the individual modal frequencies [12]. However, above $300 \mathrm{~Hz}$ all the guitars occupy a very similar range. Strikingly, throughout the range above $300 \mathrm{~Hz}$ the admittance magnitude of the banjos is typically $20-30 \mathrm{~dB}$ higher than that of all the guitars. This difference will be shown in Section 3.2 to account for the results in Figure 4. Figure 5 suggests that the particular choice of guitar makes little difference to the contrast with the banjo, especially in respect of the results in Sections 2.2 and 2.3 since they only involved playing on the top strings with all string overtone frequencies above $294 \mathrm{~Hz}$.

At first sight, the banjo results shown here conflict with the only previously published measurement of a banjo admittance, in Figure 11 of Stephey and Moore [5]. However, that earlier measurement was made with undamped strings, giving a different appearance, and furthermore the authors have confirmed that there was a calibration error by a factor $10(20 \mathrm{~dB})$. Allowing for these two effects, the measurement seems quite consistent with the one presented here.

Figures 6 and 7 show additional measurements that give a more complete characterisation of the Deering banjo.

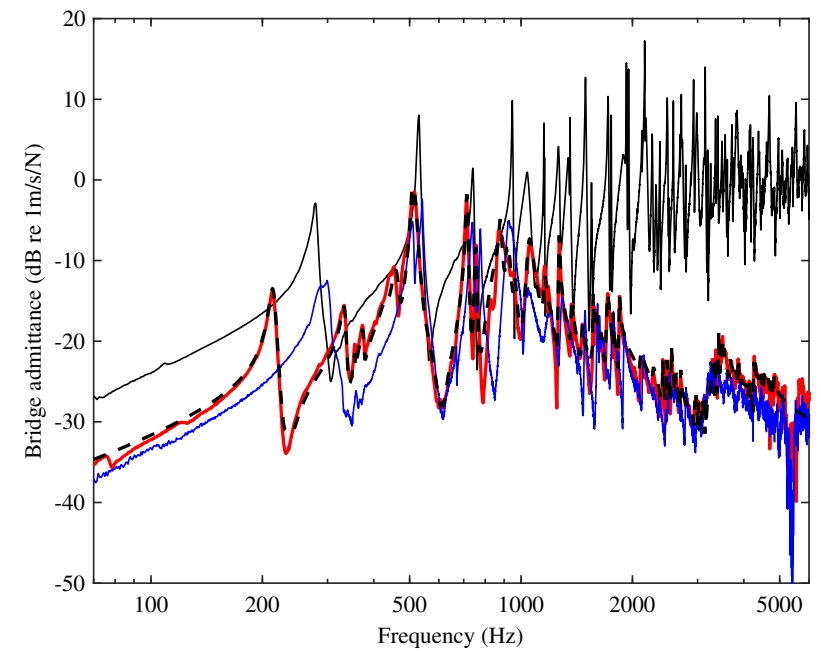

Figure 6. Admittance functions for the Deering banjo. Red: as red curve in Figure 5; black dashes: modal fit to the red curve; blue: corresponding admittance with the resonator back removed; black solid: bare head of the banjo with bridge, strings and resonator removed.

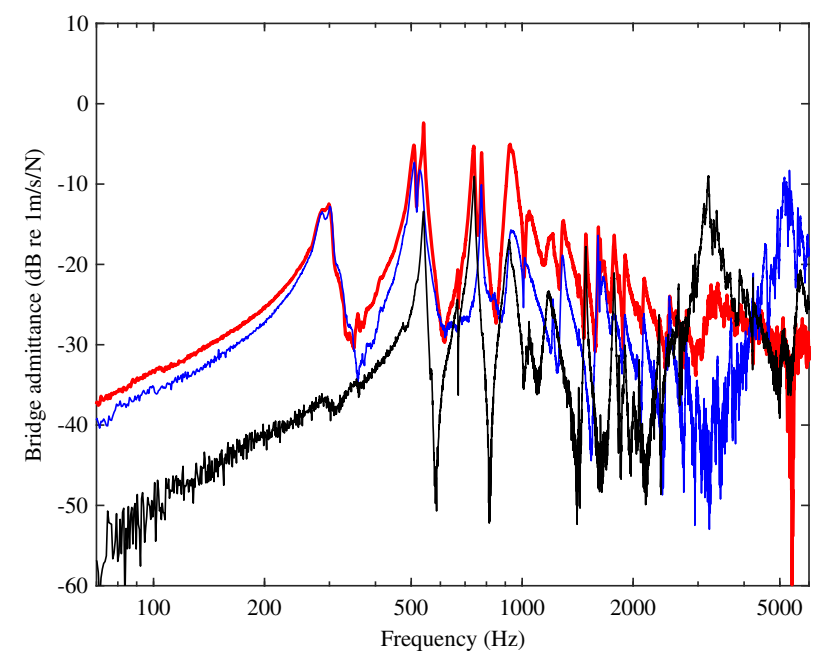

Figure 7. Admittance functions for the Deering banjo with the resonator back removed. Red: as blue curve in Figure 6; blue: measured near bridge notch for third string; black: measured on side of bridge saddle, parallel to membrane.

They immediately reveal some important features, and they will play a role in the companion paper [2] by illuminating different aspects of the underlying physics and also giving a wider range of validation cases for comparison with numerical modelling. The blue curve in Figure 6 shows the effect of removing the resonator back. The main difference from the red curve is at low frequency. As is familiar from earlier literature on the guitar (see for example [9]), and also on loudspeaker design [33], coupling to the internal air of the resonator produces an additional degree of freedom, so that the original measurement (red curve) has two peaks whereas without the resonator (blue curve) there is a single 
peak near $300 \mathrm{~Hz}$, lying between the pair. Of course, there are other more subtle changes associated with removal of the resonator: coupling to other internal acoustic resonances in the cavity, and modifying the sound radiation behaviour. The companion paper [2] gives detailed discussion of the sound radiation question.

The solid black curve shows the input admittance on the banjo head with the strings and bridge removed, at a position close to that used for the other tests. For this measurement, the resonator was also removed to give a system as close as possible to the ideal circular membrane. The measurements on the bare membrane proved quite challenging, for reasons to be explored in the companion paper [2], but for all three measurements excellent coherence was obtained up to at least $10 \mathrm{kHz}$. It is immediately clear that removing the strings and bridge from the banjo to leave the bare membrane has a very profound effect on the behaviour.

The final ingredient of Figure 6 is the dashed black curve. This shows a fitted version of the red curve, based on modal decomposition (see for example Ewins [34]). It shows an excellent level of agreement between the fitted curve and the original measurement. It will play a role when synthesis methods are discussed in Section 3.

Figure 7 shows admittance measured at two other positions on the banjo bridge, compared to the standard position near string 1. All three of these curves were obtained with the resonator back removed. The red curve shows the admittance at the position of string 1 , and is the same as the blue curve in Figure 6 . The blue curve shows the admittance measured at the bridge centre, the position of string 3 . It follows the red curve closely at the lowest frequencies, but after that the two curves diverge. Over most of the range $1-4 \mathrm{kHz}$ the blue curve is significantly below the red curve, but around $5 \mathrm{kHz}$ it rises to a conspicuous peak. The black curve shows a rather similar peak, centred around $3 \mathrm{kHz}$. This curve was obtained by tapping and measuring on the side of the bridge saddle, parallel to the head membrane. It is the admittance relevant to excitation of the head by the polarisation of string vibration lying in the plane parallel to the head.

Figure 7 gives a first glimpse of a phenomenon that will prove to be important: banjo admittance is influenced by three strong formant-like features. The first of these is a broad feature occupying the approximate frequency range 200-2000 Hz, dominating the trend of the red curve. The other two, around $3 \mathrm{kHz}$ and $5 \mathrm{kHz}$, are brought out most clearly by the black and blue curves respectively. It will be shown in the companion paper [2] that the two formants at higher frequency both have their origin in the dynamic behaviour of the banjo bridge, in conjunction with the membrane on which it sits. They are both closely analogous to the "bridge hill" of the violin: see for example [22-24]. The low-frequency formant has a quite different origin. The frequency and bandwidth of this formant are mainly determined by the bridge mass, and two sources of stiffness: one from the transverse compliance of the membrane, and the other from a combination of effects of string tension and axial stiffness of the strings and membrane, acting through

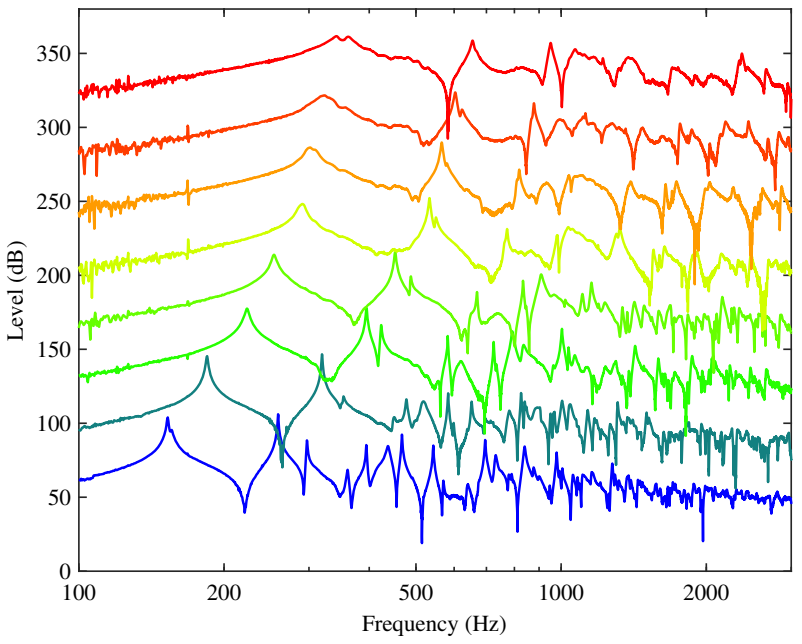

Figure 8. Results for variations of head tension: waterfall plot of the frequency response deduced from microphone recordings of head taps with eight different settings of the head tension in a banjo. Tensions are indicated by colour: blue for the lowest tension, going through the rainbow sequence to red for the highest tension. Curves are spaced by successive intervals of $40 \mathrm{~dB}$ for clarity.

the geometry of the break angle of the strings over the bridge. These effects will be explored in detail in the companion paper [2].

\subsection{Varying the head tension}

It is useful to show one more set of measured results, to illustrate the effect of varying membrane tension. A banjo with easily adjustable head tension (a Nechville Moonshine) was set to a number of different tensions, covering the full feasible range from a tension that would be too low for any modern banjo with a Mylar head (although within the range used for some drums), up to a high tension on the brink of failure of the head. In each case the sound of tapping the head with a pencil was recorded. A set of frequency spectra of these sounds is shown in Figure 8, with the lowest tension at the bottom and the highest at the top.

Increasing the tension will naturally increase the frequencies of each individual vibration mode, and a pattern of steadily rising frequencies is immediately visible. But the tension also influences the sound radiation and the associated radiation damping, as will be explained in detail in the companion paper [2]: a higher tension means a higher wave speed, so that the speed in the membrane comes closer to the speed of sound in air. Figure 8 clearly shows bandwidth increasing with tension, most obviously for the lowest mode.

Figure 9 shows the frequency and loss factor of that lowest mode for each case, plotted using the same colour code as Figure 8. Included in the plot are results from two theoretical models that will be presented in the companion paper [2]. Black symbols represent frequencies and radiation damping computed using a detailed Finite Element/Boundary Element (FE/BE) model, while 


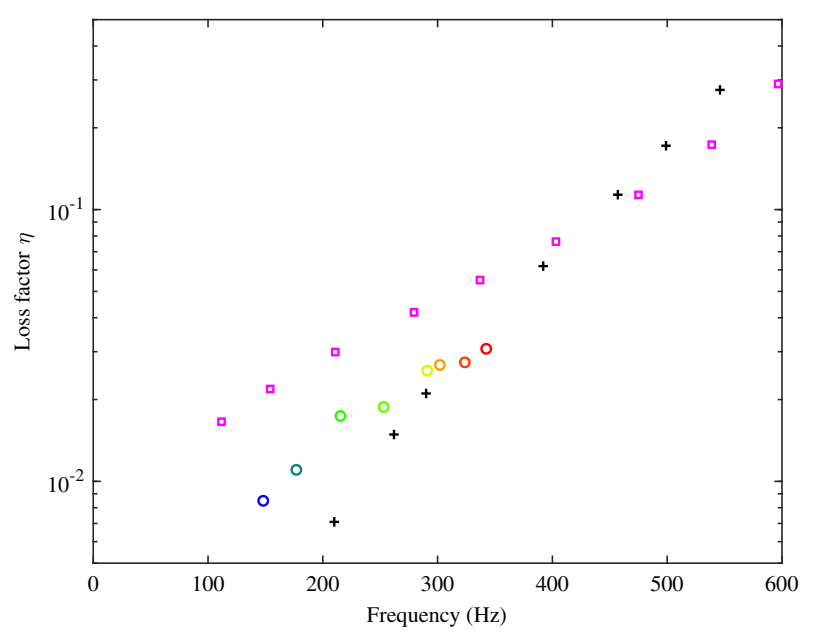

Figure 9. Results for variations of head tension: loss factor against frequency for the fundamental mode of the membrane with the eight tension settings (circles, tensions indicated by colour as in Fig. 8), compared to computed values of frequency and radiation damping from the $\mathrm{FE} / \mathrm{BE}$ model (black plus symbols) and the rectangular membrane model (magenta squares).

magenta squares were obtained from a simple model based on rectangular geometry. These two lines of points follow the general rising trend of the measured values, but deviate in detail. A major difference between the two models is that the $\mathrm{FE} / \mathrm{BE}$ calculations were for an unbaffled structure, whereas the rectangular model assumes a baffle. This highlights an issue that will become important when sound demonstrations are discussed: results will prove to be sensitive to details of damping, and vibration damping is notoriously hard to model theoretically. Measurements like these and the loss factor results seen in Figure 4 will be used to fine-tune damping models.

The theoretical results extend to far higher tensions than could safely be achieved on a real banjo. It may be noted that for the membrane wave speed to match the sound speed in air, the fundamental frequency would need to be at least doubled relative to the highest achieved in these measurements. The conclusion is that a Mylar membrane, whether on a banjo or a drum, is always subsonic.

Finally, the recordings were processed in the same way used to generate Figure 4, giving the results plotted in Figure 10. For clarity, only the extreme cases plus one intermediate tension are shown. For each tension (indicated by colour) two different taps were analysed and plotted with circle and star symbols respectively to give an indication of repeatability. As before, the magenta line indicates the approximate limit of applicability of the method: points lying much beyond this line will not be reliably detected. The results tell a clear story. For each tension there is a cloud of points representing a range of possible loss factors at any given frequency. These clouds overlap, but there is a systematic movement upwards in the diagram as tension increases. This rise in damping is caused by increasing

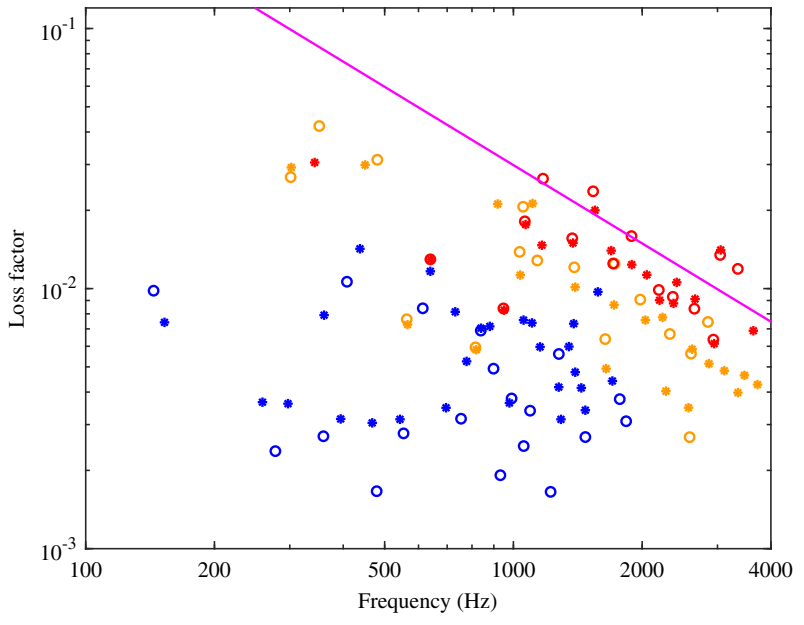

Figure 10. Loss factor against frequency for fitted modes of selected examples of the variable tension set. Tensions are indicated by colour as in Figure 8. Circles and stars show results from separate tests.

radiation damping. The conclusion for the sound of the banjo is that increasing the head tension will increase the radiation efficiency of all modes, and also change the tonal balance across the frequency spectrum by shifting individual resonances and perhaps by changing the general trend of response with frequency.

\section{Sound synthesis based on measured admittance}

\subsection{Types of synthesis model}

Measured input admittance can be used to synthesise plucked notes. Such synthesis can be used to understand the results shown in Section 2.3, and also to generate sound files for listening purposes. As has been explored in some detail in previous work $[30,35]$ there are several possible approaches to such synthesis. Two of the methods presented in that work will be used here. The first method works in the frequency domain, and uses an inverse FFT to obtain the time history of the plucked note. This method can use a measured bridge admittance with no additional processing. Alternatively, it can use a bridge admittance computed from a theoretical model.

The second method works by modal superposition. For admittance derived from theoretical modelling, the modes may be directly available from the model. For a measured admittance like the ones shown here, a modal decomposition must be carried out. This can be done using signal processing methods derived from the fields of system identification or experimental modal analysis (see for example [34]). The result of such a modal fit to a measured banjo admittance was shown in Figure 6.

The main motivation to make some use of this modalbased synthesis method is to quantify the distinction between "string modes" and "body modes". The simpler, faster and more robust frequency-domain approach cannot 
give this information. The modal-based approach uses separate degrees of freedom for the string and body, so for any given coupled mode of the combined system it is easy to compute the fraction of total potential energy associated with the string degrees of freedom. If that fraction is bigger than 0.5 the mode is labelled a string mode, otherwise it is labelled a body mode. In almost all cases the energy fraction is very close to zero or 1, making the distinction uncontroversial. The synthesised pluck sound is created by a linear superposition of all the modes, so if desired the string modes and the body modes can be separately summed before being combined to give the final sound. Sound examples will be discussed in Section 3.3.

There is a further subdivision of synthesis methods, depending on whether string vibration is confined to a single polarisation plane, or whether both polarisations are included. There is no doubt that a 2-polarisation model captures more of the physics of the instrument. Examples of the effect of including the second polarisation are included among the sound demonstrations (see Sect. 3.3), but otherwise for the purposes of this initial study only a single polarisation will be considered, normal to the "soundboard" of the instrument. This simplifies matters because only a single input admittance is required, as opposed to the $2 \times 2$ matrix of admittances required in the more general case. The results to be presented here and on the web site [3] give a strong indication that recognisable banjo sound can be produced by this simpler modelling option.

For all approaches to synthesis, properties of the strings are needed: relevant properties of guitar strings have been published previously [15], while properties of the banjo strings are listed in Table 2. The main missing ingredient is a model for the intrinsic damping of the banjo strings. A suitable model was fine-tuned by comparing the results of synthesis with measurements of real plucked notes: the details will be presented in Section 3.2.

Exploration of these varieties of synthesis model requires two stages: the first based on measurements, the second based on listening to the resulting sounds. It is a common experience in musical acoustics that features which show up very clearly in acoustical measurements do not necessarily produce large audible effects, while conversely some effects that are perceptually important can be remarkably hard to pin down by measurements.

\subsection{Model validation: distribution of loss factors}

It has been seen in Figure 5 that the bridge admittances of guitars and banjos are significantly different. To see how this translates into different behaviour when the instruments are played in the normal way, the distribution of loss factors shown in Figure 4, deduced from playing chromatic scales on the top strings of the banjo and guitar, can be compared with estimates derived from modelling. The measured clouds are plotted again in Figure 11 for the guitar and the banjo separately: two sets of plucked notes were recorded for each instrument, and processed independently to give an indication of consistency of the measurements:
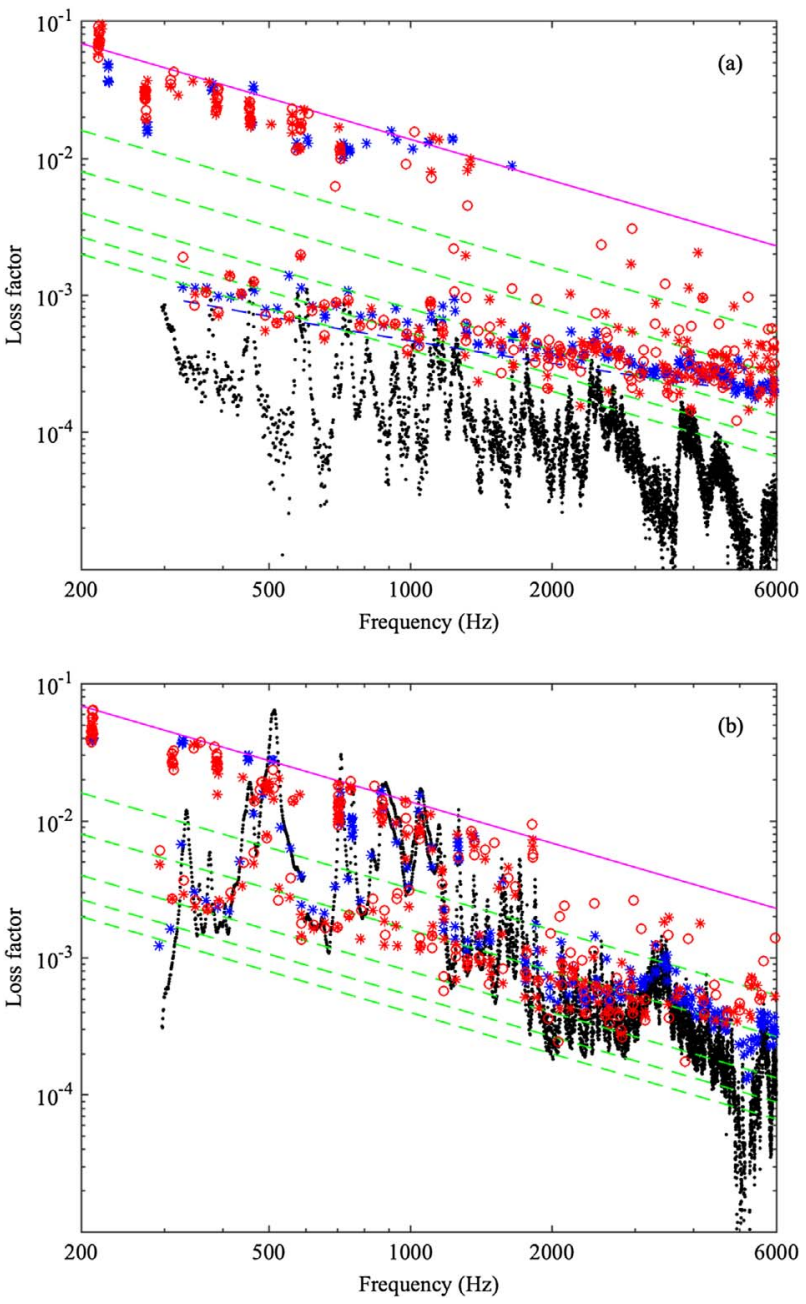

Figure 11. Loss factor versus frequency for modes excited by plucking the top string of (a) the guitar; (b) the banjo. Red circles and stars: measured values; blue stars: values from synthesised notes; black dots: predicted loss factor for energy flow into the body alone, calculated from the measured admittances shown in Figure 5. The analysis method cannot detect modes significantly above the magenta line. Green dashed lines indicate decay time constants $(\omega \eta)^{-1}=50 \mathrm{~ms}$ (top line), $100 \mathrm{~ms}, 200 \mathrm{~ms}, 300 \mathrm{~ms}$ and $400 \mathrm{~ms}$ (lowest line). Blue dashed line in (a) shows the internal loss factor of the string.

the two sets are plotted as red circles and stars, and a reassuring correspondence can be seen between the two over the important parts of both plots.

The blue and black points in these plots show theoretical estimates of two different kinds. The black points show estimates of the contribution to the loss factor arising only from energy flow from the string into the instrument body, calculated from the measured bridge admittance. The reflection coefficient, $R(\omega)$, for a transverse wave on the string incident on the bridge can be expressed in terms of the admittance $Y_{b}(\omega)$ :

$$
R(\omega)=\frac{Y_{b}(\omega)-Y_{0}}{Y_{b}(\omega)+Y_{0}}
$$


where $Y_{0}$ is the characteristic admittance for the string, the inverse of the impedance listed in Table 1 (see for example Cremer [36]). The loss factor $\eta_{b, n}$ associated with dissipation during this incomplete reflection for the $n$th overtone of string vibration depends only on the real part of $R^{2}$ : it can be written

$$
\eta_{b, n} \approx \frac{1-\operatorname{Re}\left(R^{2}\right)}{2 \pi n}
$$

and provided the ratio $Y / Y_{0}$ is small in magnitude this reduces to

$$
\eta_{b, n} \approx \frac{2 \operatorname{Re}(Y)}{\pi n Y_{0}}
$$

Using the measured bridge admittance of the guitar and the banjo, respectively, this loss factor has been calculated at intervals of $1 / 30$ semitone for all overtones falling within the frequency range, and plotted as the black symbols in Figure 11.

The blue points in Figure 11 are the result of repeating the chromatic scale experiment using the frequency-domain synthesis model, and processing the results with the identical analysis code used for the experimental observations (so they are again limited to the region below the magenta lines). These points can be directly compared to the measured red points, and they also serve to give an internal check both on the synthesis code and on the signal processing method, by comparing with the black points. Both blue and black points are computed, by very different approaches, using the same bridge admittance, so the blue points should always lie above the black points because the synthesis model allows for additional energy loss in the string. But this additional loss is quite small, so when the black points predict relatively high loss factor, the blue points would be expected to follow them. These expectations are reassuringly confirmed in the plots, most clearly in the banjo case.

The plots tell an interesting story. It is simplest to explain the guitar case first, Figure 11a. The majority of the red and blue points mark out two lines, one with loss factors of the order of $10^{-2}$ and the other of the order of $10^{-3}$. As already pointed out, these indicate "body modes" and "string modes" respectively. The body modes often show as clusters of many points, because in principle these modes are excited by the transient nature of every plucked note, regardless of the played pitch, so that many estimates of these modes are obtained from the chromatic scale. Strictly, each body mode is not exactly the same for every played note, because it is perturbed by coupling to the string. However, except for special cases where a string overtone falls very close to an unperturbed body mode, the shift is small. The blue and red points agree quite well for these body mode clusters. Probably the line of points would continue approximately horizontal beyond the magenta line but for the limitations of the analysis technique.

The string modes consist of an approximately harmonic series based on the fundamental of each successive note so that the plotted points are spread out along the frequency axis. The body modes have Q-factors (inverse of loss factors) around 100 or lower, while the string modes have Q-factors of a few thousand and their decay times determine the duration of each played note. It is very striking in Figure 11a that the line of the string modes is fairly featureless, and mostly lies significantly above the black points. This confirms something reported earlier for guitars (it is true for both steel-string and nylon-string guitars) [37]: rather unexpectedly, the decay rate of string modes in these instruments is dominated by the damping of the string itself, and loss into the body of the instrument is usually only a small perturbation. There are exceptions where the guitar body has a strong resonance, but it should be noted that this particular plot is confined to the frequency range relevant to the top string, and the strongest body resonances of the guitar lie lower in frequency.

The blue points for string modes follow the red points closely, but this is no coincidence: this data was used to fine-tune the damping model for the string used in the synthesis model. Earlier work investigating a range of polymer-based musical strings demonstrated that a rather simple model for the intrinsic damping of the strings gave excellent agreement with measurements [38]. A version of that damping model has been successfully adapted for the steel strings played here: the loss factor resulting from this model is shown as the dashed blue line in Figure 11a. The total modal loss factor is estimated by

$$
\eta_{n} \approx \eta_{\text {background }}+\eta_{\text {air }}+\eta_{\text {bend }}
$$

in which a constant "background loss factor" $\eta_{\text {background }}=$ $3 \times 10^{-5}$ is added to a term associated with damping from viscosity in the surrounding air, and a term associated with bending stiffness of the string. The air damping term is given by Fletcher and Rossing [19] in the form

$$
\eta_{\text {air }} \approx \frac{\rho_{a}}{\rho} \frac{2 \sqrt{2} M+1}{M^{2}},
$$

where $\rho_{a}$ is the density of air, and

$$
M=\frac{d}{4} \sqrt{\frac{2 \pi f_{n}}{\eta_{a}}},
$$

where $\eta_{a}$ is the kinematic viscosity of air. Textbook values are used: $\rho_{a}=1.2 \mathrm{~kg} / \mathrm{m}^{3}$ and $\eta_{a}=1.5 \times 10^{-5} \mathrm{~m}^{2} / \mathrm{s}$. Energy loss from viscoelasticity in the string arises from the influence of bending stiffness. If the Young's modulus of the string has the complex value $E\left(1+i \eta_{E}\right)$, an argument based on Rayleigh's principle [39] can be used to yield an expression for the associated loss factor of the $n$th string mode:

$$
\eta_{\text {bend }} \approx \frac{E \pi^{2} d^{2} n^{2} \eta_{E}}{64 \rho L^{4} f_{1}^{2}+E \pi^{2} d^{2} n^{2}},
$$

where the string has vibrating length $L$, diameter $d$, density $\rho$ and fundamental frequency $f_{1}$. This bending term turns out to have only a minor influence for plain 
steel strings over the frequency range examined here: the value $\eta_{E}=10^{-3}$ was used, but the exact value does not matter very much.

The plot for the banjo, Figure 11b, is strikingly different from the guitar case. The black points lie considerably higher over much of the frequency range, as a direct result of the higher input admittance of the banjo. There is still a trace of two lines showing string modes and body modes, but whenever the black curve crosses above the position where the line of string modes occurred for the guitar, it carries the actual loss factors up with it. Energy dissipation arising from different physical mechanisms is additive, so in theory the total loss factor cannot be lower than the black curve.

In the main this expectation is borne out by the data. There are a few red points lying below the black curve, especially in the frequency range around $1 \mathrm{kHz}$, but these may be associated with an aspect of the physics not taken into account in this simple description: each string mode can occur in two different polarisations. The description here, and the basis for the calculation of the black points, considers only the string polarisation normal to the head. Vibration in the plane parallel to the head is likely to couple much less strongly to the head, and thus exhibit lower loss factors. The real plucks during the test procedure will involve a mixture of both polarisations, this being the explanation of the beating behaviour noted in Figure 2b. Perhaps a few peaks associated with the second polarisation have been caught by the analysis. Simple experiments using the two-polarisation synthesis method confirm that this kind of effect can indeed occur, but it would require more careful and systematic efforts to attempt a quantitative validation comparison. This lies beyond the scope of the present work.

There are several consequences for the behaviour of notes played on the banjo. For frequencies up to about $1 \mathrm{kHz}$, the string modes often have significantly higher damping than for the same string attached to a guitar. The decay time will be faster, and at some frequencies it will be so fast that the distinction between string modes and body modes is lost: this is flagged in the plot by the black points reaching levels comparable with the line of body modes. Any note with a fundamental or low overtone lying in one of these frequency ranges might be perceived by a player as "falling flat": it will not ring on as much as usual. Secondly, over most of the frequency range plotted here the string modes follow the black points quite closely. This means that most of the energy put into the string is lost by being transferred to the body, whereas in a guitar most of it is dissipated by other loss mechanisms. Furthermore, it will be shown in the companion paper [2] that radiation damping for many modes of the banjo head dominates over structural damping, so a significant proportion of this energy from the strings is radiated as sound.

The result of these two factors taken together is that a note played on the banjo with the same player gesture as a note on the guitar will sound louder, and decay faster. No player is likely to quarrel with that description, and it is at least part of the essence of "banjoness". But there are probably other important factors, not made apparent by this particular analysis. Physical measurements are never enough to settle perceptual questions: it is necessary to listen to the sounds from the synthesis model and find out if they do in fact strike listeners as convincingly banjo-like.

\subsection{Synthesised sound examples}

To accompany this discussion, the reader is referred to Section 5.5 of the web site [3]. All the sound examples use the same short musical passage, the first few measures of a banjo arrangement of the tune "The Arkansas traveler". The first example appears here and as Sound B.1 on the web site. It makes use of the measured admittance in the red curve of Figure 5, and it is the crucial first test of whether the synthesis method can give plausible banjo sounds. This can be compared with sounds based on other measured admittances from this banjo, in Sounds C.1-C.8 on the web site. These include cases with and without the resonator back, cases measured at different points on the bridge, and cases in which the regular bridge was substituted for a rigid circular bridge. (This circular bridge is pictured on the web site; it will be used in the companion paper [2] as a validation case for modelling.)

In the opinion of the authors, these cases all "sound like banjos", although they sound different from each other in detail. This contrasts with Sounds C.9 and C.10, which use the measured admittances of two different guitars, and Sound C.11, which uses the admittance of a violin. The difference is striking. It seems unlikely that any listener would mistake any of these sounds for a banjo.

Sounds E.1-E.4 illustrate the use of a two-polarisation synthesis model, using a measured $2 \times 2$ admittance matrix. Three different values of the plucking angle $\theta=0^{\circ}, 45^{\circ}, 90^{\circ}$ are illustrated. The last of these sounds the most distinctive, which is not surprising given that it will be dominated by the horizontal bridge admittance plotted in black in Figure 7. Sound E.4 also exhibits a phenomenon to be discussed shortly: there is an unrealistic "zinginess" accompanying the sound.

Sounds E.5-E.7 relate to the modal synthesis approach. They give an opportunity to hear the separate sounds of the string (1) and body modes making up the full synthesised sound. This comparison gives a useful insight. On a casual listening, the string-only sound is quite similar to the full synthesis with all modes, which is indistinguishable from the frequency-domain datum sound presented above. However, the difference is clearly audible, if hard to put into words.

Figure 12 shows spectrograms of the synthesised passage for the string modes and the body modes separately. They reveal that it is hardly surprising that some difference can be heard when the body modes are included. Body modes are strongly excited over a frequency range extended up to about $2 \mathrm{kHz}$, and although they generally have much faster decay times than the string modes, typical banjo music like the passage used here has notes that come thick and fast, and the body modes ring on for long enough to bridge the gap to the next played note. They surely make 

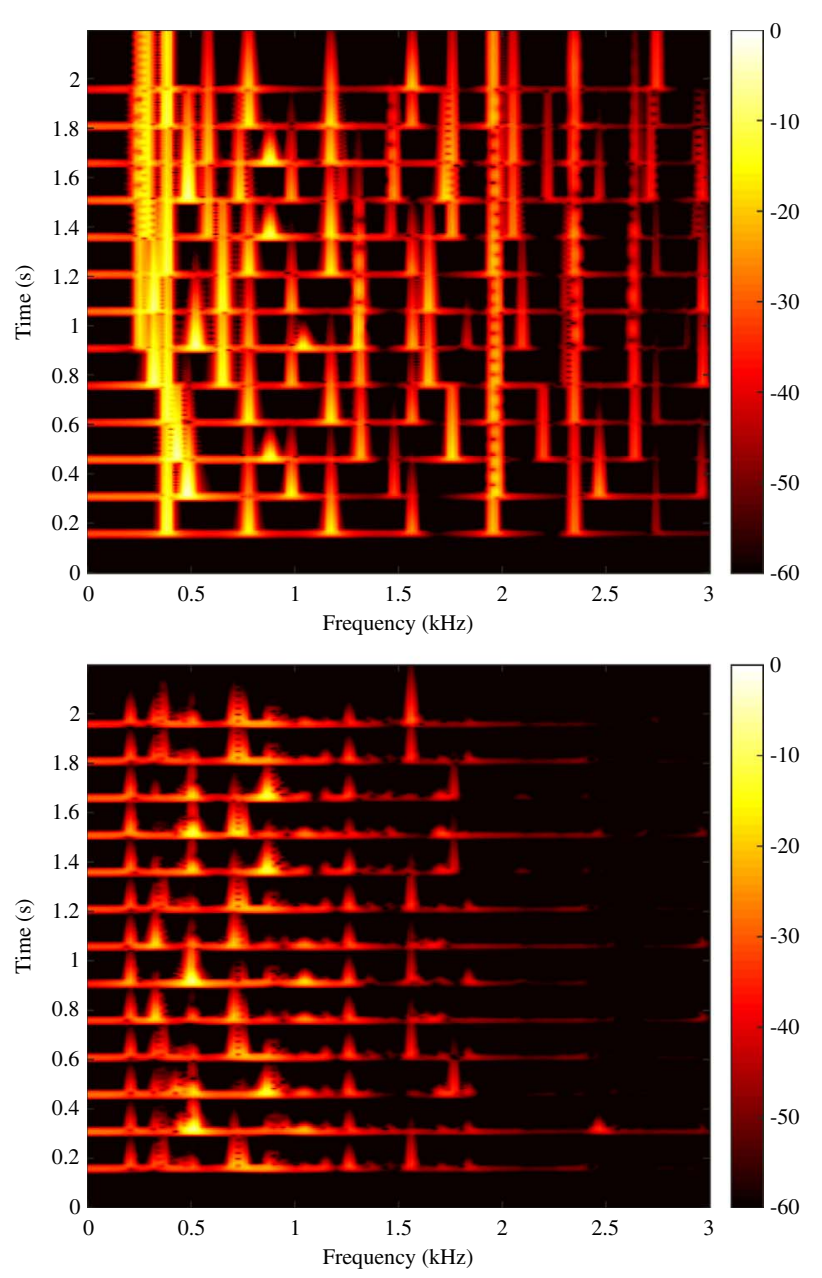

Figure 12. Spectrograms to illustrate modal synthesis: (a) string modes only; (b) body modes only.

a significant contribution to the "punctuation" at the start of each note, and it seems likely that this effect forms a significant part of characteristic banjo sound.

Note that this body mode contribution to the sound gives a possible mechanism for recognising a particular instrument, to some extent independent of what music is played. The mix of body modes is similar for every note, and constitutes a kind of acoustical fingerprint of the instrument. A similar mechanism has been suggested for distinguishing different violins by their transient sound [40]. Fréour et al. [31] have studied the audibility of comparable body sounds in guitar notes, with the conclusion that the effect is confined to frequencies below about $1 \mathrm{kHz}$ and is strongest for the low modes below $300 \mathrm{~Hz}$, in keeping with the impression given by Figure 2b. For the banjo, Figures $2 \mathrm{a}$ and 12 suggest that the effect may extend over a wider frequency range, but this has yet to be formally tested.

The next set of sound demonstrations, Sounds F.1-F.10 and G.1-G.13, are computed on a different basis. Rather than using measured admittance, they use a theoretical model in order to give access to parametric variations.
The model used is a deliberately crude one, in order to explore the hypothesis that anything with modal density and sound radiation behaviour that is membrane-like rather than plate-like can "sound like a banjo". So rather than using the detailed FE/BE model mentioned earlier, these sound examples are based on a rectangular membrane: the details of the model development and parameter selection are described in the companion paper [2]. The datum case for this model appears here and as Sound B.2 on the web site, to be compared directly with Sound B.1 described earlier, based on measured admittance.

The various sound examples illustrate the effect of varying the size and tension of the banjo head, and also the mass of the bridge and the added stiffness due to axial effects in the strings, in-plane effects in the head and string tension. The details are described on the web site. An important conclusion emerges, illustrated by the admittance plots in Figure 13. Adjustments that affect individual resonances but which do not change the low-frequency formant, like the examples shown in Figure 13a, have a relatively subtle effect on the sound, whereas adjustments that change this formant, like those shown in Figure 13b, have a more pronounced effect. Here are the sounds corresponding to the blue (1) and black (1) curves in Figure $13 \mathrm{a}$, and here are the sounds corresponding to the red (1)) and black (1) curves in Figure 13b. Examples like these provide the basis of the claim made earlier that this banjo formant appears to have higher perceptual salience than individual modes. This hypothesis deserves to be tested in a formal psychoacoustical study.

The final set of demonstrations, Sounds H.1-H.5, illustrate the influence of the formant near $3.5 \mathrm{kHz}$, seen in Figure 7 . Weighted mixtures of the measured admittance and the simulated admittance from the rectangular model were computed, and the crossover frequency between them was varied so that the formant could either be excluded or included.

Certain of the sound examples exhibit a phenomenon that strikes many listeners as unrealistic. The effect was first noticed in the context of synthesis using the model based on rectangular membrane geometry, but it also occurs in some cases based on measured admittance: Sounds C.5 and E.4 give clear examples. These cases are all characterised by a sense of "something ringing on too long" in an unrealistic way, and the effect apparently arises from damping that is too low in the mid-kHz range. Details of the problem and the measures taken to minimise its effects by adjustments to damping models are given on the web site [3].

There is an intriguing possibility that deserves to be mentioned here. The effect is very persistent, so perhaps it is a real phenomenon, but one that is not obvious from normal banjo playing. It should be recalled that what is computed by the synthesis algorithm is not the radiated sound, but the motion of the body at the bridge. Different modes of the banjo head have very different levels of radiation damping (see for example Fig. 7 of the companion paper [2]). Modes with low radiation damping, and thus 

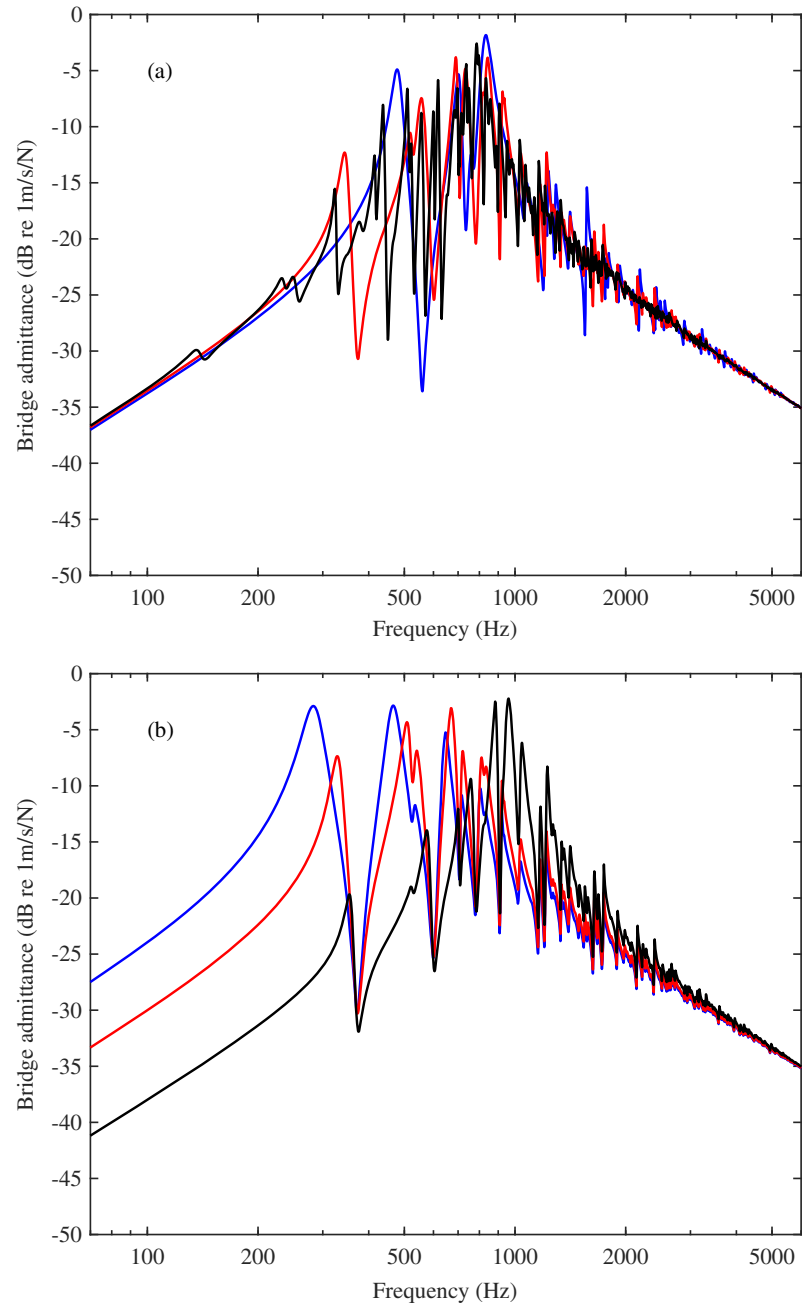

Figure 13. (a) Admittances calculated from the rectangular membrane model, resulting from varying head size by factors 0.75 (blue), 1 (red), 2 (black), corresponding to Sounds F.2, F.3 and F.5 respectively; (b) similar admittances from varying string axial stiffening with values 0 (blue), $10 \mathrm{kN} / \mathrm{m}$ (red), $40 \mathrm{kN} / \mathrm{m}$ (black), corresponding to Sounds G.8, G.10 and G.13 respectively.

high $\mathrm{Q}$ values, presumably do not feature very strongly in the sound received at a distance from the banjo, but they may be strongly present in the body motion. In principle this effect could be allowed for in synthesis using the theoretical model, but it would rely on using the modal approach to synthesis. Synthesis direct from measured admittance can only be done in the frequency domain, so the option to weight the modes differently according to their radiation efficiency is not available.

This idea would suggest that a real banjo might sound rather harsh if some kind of body pickup was used to allow amplified sound. It might also sound harsh in a recording using a close microphone, which would pick up near-field sound from modes with low radiation efficiency. Both predictions are consistent with anecdotal evidence about amplifying and recording banjos. So perhaps the banjo, with very low intrinsic damping in both strings and body, really is on the edge of making unpleasant sounds like those heard in some of the synthesised files.

\section{Discussion and conclusions}

In this paper and its companion [2], together with an associated web site [3], the physical acoustics and corresponding perceptual qualities of a 5-string American banjo have been explored. The resulting models have been calibrated and validated by extensive measurements. These measurements and models have then been used to generate a range of sound files to illustrate the perceptual effects of the predictions, including the effects of varying each of the main parameters. No formal psychoacoustical investigations have been carried out here, but based on informal listening to these sound files it is now possible to suggest some answers to the various general questions raised in the Introduction. Testing these suggestions is a topic for future work.

The first question is whether it is possible to produce convincingly banjo-like sounds using linear synthesis models. Based on the examples presented here, the answer seems to be affirmative. The most important ingredient needed to achieve distinctive banjo sound seems to be the decay rate of "string modes", which is significantly and systematically higher for the banjo than for a guitar: see Figure 11. One sound example was given in which only the string modes were included, and this is already recognisably banjo-like. Other factors contribute to the perception of characteristic banjo sound, but this sound file suggests that their influence may be secondary.

The faster decay rates occur because bridge admittance of a banjo is significantly higher than that of a guitar over most of the relevant frequency range (see Fig. 5). The main reasons are the light weight of the banjo membrane compared to a guitar soundboard, and the higher modal density of a membrane-based system compared to any plate-based structure. A familiar attribute of banjo sound follows. For a given initial excitation of the string by the player, banjos give louder radiated sound than guitars, particularly in the early part of each note (see Fig. 3). The closer impedance match between strings and body gives part of the explanation, but it is not the whole story.

Measurements reveal that the structural component of damping of a banjo membrane is very small, perhaps an order of magnitude smaller than the corresponding structural damping contribution in a wooden plate like the soundboard of a guitar. So, especially at lower frequencies up to the low $\mathrm{kHz}$ range, damping factors for at least some modes of the banjo membrane are dominated by radiation damping. Those modes are automatically responsible for high levels of radiated sound, with obvious consequences for the loudness of the banjo.

For any instrument, the action of plucking a string provides step-function excitation of the body modes as well as the string modes. Following the release, there will be a transient sound from those body modes. The effect is present in the guitar, but the audible effect is largely 
confined to a small number of low-frequency modes. In the banjo, with stronger coupling between string and body, this effect is larger in amplitude and also extends to higher frequencies, assisted by the increasing modal density of the banjo membrane.

When the bridge and strings are added to a banjo membrane, three formants are created which shape the envelope of the admittance. A low-frequency formant governs the response in the frequency range up to about $2 \mathrm{kHz}$. Analysis in the accompanying paper [2] reveals that the frequency and bandwidth of this formant are mainly determined by the bridge mass, and an effective stiffness acting at the bridge. This stiffness is associated with the axial stiffness of the strings, in-plane stiffness of the head, and an effect of string tension. All these factors are sensitive to the break angle of the strings over the bridge. Parameters that influence this formant, such as the bridge mass or the break angle, can produce large perceptual effects. By contrast, parametric changes which affect individual modal frequencies but leave the formant unchanged seem to produce only rather subtle changes to perceived sound. This contrasts with the situation for the guitar, where individual low modes are very important for perceptual discrimination.

At higher frequencies, with the particular pattern of bridge relevant to this study, two "bridge hills" were seen. These are governed by dynamic behaviour of the bridge, strongly influenced by the membrane beneath the bridge feet. The phenomenon is similar to one that has been widely studied in the violin [23, 24]. One of these hills, at about 3 $\mathrm{kHz}$, mainly affects the outer strings of the banjo, while the second hill, at around $5 \mathrm{kHz}$, has its biggest influence on the middle string. These hills seem to be the main cause of differences in the admittance at the different string notches. The result is a significant influence on the sound, among other things confirming the opinion of players that the 3rd string of the banjo can sound very different from the outer strings.

Banjo players will commonly adjust the tension of the head membrane to influence tonal qualities. Increasing tension raises resonant frequencies, of course, but it also systematically increases radiation damping by bringing the wave speed in the membrane closer to the speed of sound in air. The result may be to increase loudness, although an increase in radiation efficiency may be offset by a reduction in average admittance from the higher tension. There is also likely to be a change in the tonal balance of the instrument. It is reassuring to report that these trends are all familiar to banjo adjusters: tightening the head into the range typical for bluegrass banjos is generally perceived to make the sound sharper, louder and brighter, but further tightening can "choke" the sound before the point of failure is reached.

The set of sound demonstrations on the accompanying web site [3] highlights an issue that calls for further study. It seems that physics-based synthesis models for banjo sound require great care over the modelling of damping. Theoretically-based models, and even some cases based directly on measured bridge admittance, sometimes exhibit an unrealistic sound quality which seems to be associated with low damping in the $\mathrm{kHz}$ frequency range. An important illustration of this is given by the sound examples based on the detailed FE/BE model: although that model has been shown to capture many aspects of the physics very well, it does not at present give very good-sounding syntheses. This general issue of unrealistic sound associated with low damping is very persistent. It probably goes beyond simple inaccuracies in damping modelling, and has a physical basis in the acoustical properties of real banjos.

\section{Acknowledgments}

Many people have contributed to this study: the authors thank Julien Legault, Vincent Cotoni, Robin Langley, Evan Davis, Brian Moore, Thomas Moore, Martin Woodhouse, Alan Heaver, and the Deering Banjo Company. We also thank three reviewers for comments leading to significant improvements.

\section{Conflict of interest}

The authors declare that they have no conflict of interests.

\section{Data availability statement}

This article includes selected audio files embedded in the article. The complete set of audio files and accompanying discussion for this article are available at https:// euphonics.org/ Chapter 5, Section 5.5.

\section{References}

1. J. Woodhouse, R.S. Langley: Interpreting the input admittance of violins and guitars. Acta Acustica United with Acustica 98, 4 (2012) 611-628.

2. J. Woodhouse, D. Politzer, H. Mansour: Acoustics of the banjo: Theoretical and numerical modelling. Acta Acustica 5 (2021) https://doi.org/10.1051/aacus/2021008.

3. Euphonics: The science of musical instruments. [Online] Available at: https://euphonics.org/ [Accessed: Dec 29 2020].

4. J. Dickey: The structural dynamics of the American fivestring banjo. Journal of the Acoustical Society of America 114 (2003) 2958-2966.

5. L.A. Stephey, T.R. Moore: Experimental investigation of an American five-string banjo: Journal of the Acoustical Society of America 124 (2008) 3276-3283.

6. J. Rae, in The Science of String Instruments, T.D. Rossing, Editors, New York, Springer-Verlag. 2010, pp. 59-75.

7. G. Derveaux, A. Chaigne, P. Joly, E. Bécache: Time-domain simulation of a guitar: model and method. Journal of the Acoustical Society of America 114 (2003) 3368-3383.

8. T.J.W. Hill, B.E. Richardson, S.J. Richardson: Acoustical parameters for the characterisation of the classical guitar. Acta Acustica United with Acustica 90, 2 (2004) 335-348.

9. O. Christensen, B.B. Vistisen: Simple model for lowfrequency guitar function. Journal of the Acoustical Society of America 68 (1980) 756-766. 
10. M.J. Elejabarrieta, A. Ezcurra, C. Santamaría: Coupled modes of the resonance box of the guitar. Journal of the Acoustical Society of America 111 (2002) 2283-2292.

11. M.K. Lee, M.H. Fouladi, S.N. Namasivayam: Mathematical modelling and acoustical analysis of classical guitars and their soundboards. Advances in Acoustics and Vibration (2016) 6084230. https://www.hindawi.com/journals/aav/ 2016/6084230/.

12. E.B. Davis: On the structural and acoustic design of guitar soundboards. Thesis, University of Washington, 1990.

13. M. Mihălcică, M.D. Stanciu, S. Vlase: Frequency response evaluation of guitar bodies with different bracing systems. Symmetry 12 (2020) 795 .

14. H.A.K. Wright, B.E. Richardson: Psychoacoustical evaluation of synthesised guitar tones, in ICA 95 - Proceedings of the 15th International Congress on Acoustics, Vol. III. 1995, pp. 521-524. https://www.icacommission.org/proceedg.html

15. J. Woodhouse, E.K.Y. Manuel, L.A. Smith, A.J.C. Wheble, C. Fritz: Perceptual thresholds for acoustical guitar models. Acta Acustica United with Acustica 98, 3 (2012) 475-486.

16. H. Mansour, V. Freour, C. Saitis, G. Scavone: Postclassification of nominally identical steel-string guitars using bridge admittances. Acta Acustica United with Acustica 101 (2014) 394-407.

17. S. Carcagno, R. Bucknall, J. Woodhouse, C. Fritz, C.J. Plack: Effect of back wood choice on the perceived quality of steel-string acoustic guitars. Journal of the Acoustical Society of America 144 (2018) 3533-3547.

18. J.W.S. Rayleigh: The Theory of Sound. Macmillan and Co., London, 1877.

19. N.H. Fletcher, T.D. Rossing: The physics of musical instruments, 2nd ed. Springer, New York, 1998.

20. A. Chaigne, J. Kergomard: Acoustics of Musical Instruments. Springer Verlag, New York, 2013.

21. B. Elie, F. Gautier, B. David: Macro parameters describing the mechanical behavior of classical guitars. Journal of the Acoustical Society of America 132 (2012) 4013-4024.

22. E. Durup, E.V. Jansson: The quest of the violin bridge-hill. Acta Acustica United with Acustica 91, 2 (2005) 206-213.

23. E. Jansson, B. Niewczyk: On the acoustics of the violin: Bridge hill or body hill? Journal of the Catgut Acoustical Society 3, 7 (1999) 23-27.

24. J. Woodhouse: On the "bridge hill" of the violin. Acta Acustica United with Acustica 91, 1 (2005) 155-165.
25. Deering Banjo Company. [Online] Available at: https:// www.deeringbanjos.com/ [Accessed: Dec 29 2020].

26. Martin Woodhouse Guitars. [Online] Available at: http:// www.woodhouse-guitars.co.uk/ [Accessed: Dec 29 2020].

27. B.C.J. Moore, B.R. Glasberg, A. Varathanathan, J. Schlittenlacher: A loudness model for time-varying sounds incorporating binaural inhibition. Trends in Hearing 20 (2016) $1-16$.

28. B.C.J. Moore, M. Jervis, L. Harries, J. Schlittenlacher: Testing and refining a loudness model for time-varying sounds incorporating binaural inhibition. Journal of the Acoustical Society of America 143 (2018) 1504-1513.

29. B.C.J. Moore: An introduction to the psychology of hearing, 6th ed. Academic Press, Amsterdam; Boston, 2013.

30. J. Woodhouse: Plucked guitar transients: Comparison of measurements and synthesis. Acta Acustica United with Acustica 90, 5 (2004) 945-965.

31. V. Fréour, F. Gautier, B. David, M. Curtit: Extraction and analysis of body-induced partials of guitar tones. Journal of the Acoustical Society of America 136 (2014) 1385-1393.

32. K.G. McConnell, P.S. Varoto: Vibration Testing: Theory and Practice, 2nd ed. John Wiley \& Sons, Hoboken, NJ, 2008.

33. W.M. Leach: Introduction to Electroacoustics \& Audio Amplifier Design, 4th ed. Kendall Hunt, Dubuque, Iowa, 2009.

34. D.J. Ewins: Modal Testing : Theory, Practice, and Application. Mechanical engineering research studies Engineering dynamics series, 2nd ed. Research Studies Press, Baldock, 2000.

35. J. Woodhouse: On the synthesis of guitar plucks. Acta Acustica United with Acustica 90, 5 (2004) 928-944.

36. L. Cremer: The Physics of the Violin. MIT Press, Cambridge, MA, 1984.

37. J. Woodhouse: Influence of damping and nonlinearity in plucked strings: Why do light-gauge strings sound brighter? Acta Acustica United with Acustica 103, 6 (2017) 1064-1079.

38. J. Woodhouse, N.J. Lynch-Aird: Choosing strings for plucked musical instruments. Acta Acustica United with Acustica 105 (2019) 516-529.

39. M.E. Mcintyre, J. Woodhouse: Influence of geometry on linear damping. Acustica 39, 4 (1978) 209-224.

40. J. Woodhouse: On recognising violins: Starting transients and the precedence effect. Catgut Acoustical Society Newsletter 39 (1983) 22-24.

Cite this article as: Woodhouse J, Politzer D \& Mansour H. 2021. Acoustics of the banjo: measurements and sound synthesis. Acta Acustica, 5, 15 . 